\title{
INTERVIEW WITH OUSSAMA M. WAZNI, MD
}

\section{ANGELINA WAGNER}

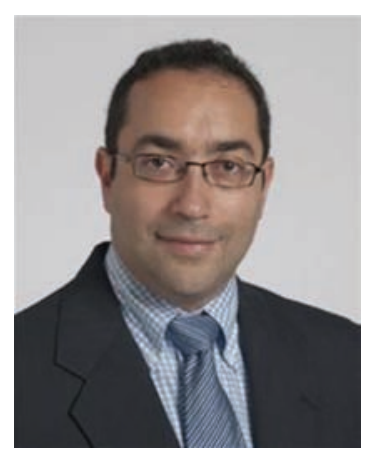

\section{Introduction}

Device infections have increased among the patient population with cardiac implantable electronic devices (CIEDs). The strategy of device lead abandonment at the time of a system upgrade or change is undergoing scrutiny based on the long-term implications: infection, clinical outcomes, and procedural success rates.

Lead abandonment remains controversial because although it avoids the risks of lead extraction, it results in a higher burden of leads and complicates future clinical outcomes especially if patients eventually require lead extraction because of complications from lead abandonment. There are no randomized controlled trials of lead extraction versus lead abandonment but there has been evidence from observational, cohort, and registry studies. These studies suggest that the potential future benefit of lead extraction outweighs the risks of lead abandonment.

Dr. Oussama M. Wazni is Professor of Medicine, Cleveland Clinic Lerner College of Medicine, and Director, Outpatient Clinic, Cardiac Electrophysiology at the Cleveland Clinic. He is the author of over 100 publications in indexed journals. Dr. Wazni has received several honors and awards, and has presented research findings at national and international meetings. He is a member of several professional organizations and societies, and also serves on the Editorial Board of several electrophysiology and cardiology journals. Dr. Wazni's active research interests include atrial fibrillation and ventricular tachycardia ablation, device implantation, and extraction.

We are speaking to Dr. Oussama M. Wazni about the risk versus risk equation of extracting redundant leads versus the long-term implications of lead abandonment.

\section{Wagner: What are the controversies regarding management of superfluous leads?}

Wazni: There is still a lot of controversy regarding leads that would become superfluous. Should a lead that we encounter that's going to become superfluous be extracted or abandoned? We face this scenario in a patient that needs an upgrade from a PPM [pacemaker] to an ICD [implantable cardioverter-defibrillator] or when a lead becomes dysfunctional. This is a controversy or an issue that we've been dealing with for many years now. If a lead is abandoned, the patient is then not subjected to the immediate risk of extraction; on the other hand, an abandoned lead may cause a lot of problems in the future and may end up exposing the patient to higher risks when this lead needs to be extracted in the future as would happen with infection.

\section{Wagner: How does the practice of lead abandonment complicate the overall management of CIED infections?}

Wazni: Suppose a patient had an abandoned lead because in the past a physician decided not to extract the lead for a variety of reasons and accomplished what they needed at that time. So now years have passed and this device is infected. With infection, all leads should be removed including the now much older abandoned ones. Because this lead had been abandoned, it is much older and with age it's a lot more difficult to extract. This is where our study comes into the picture. In this study, we showed that abandoned leads because they are older, complicate the management of device infections. The way they complicate the management is that because it's difficult to extract, there are more complications. Also, more pieces and parts of the lead are left behind. Since this is an infected device, any remnants that are left behind are thought to result in clinical failure. It is a clinical failure because in order to clear the infection all lead material should be removed from the patient. Any remnants that are left in the patient, make it more difficult for us to clear the infection.

Wagner: Could you please talk in more detail about the Cleveland Clinic study and what changes have been seen in patient management for patients with CIEDs?

Wazni: In our retrospective study, we looked at 1,386 patients from 1996 to 2012 . We grouped them into two groups: one group of about 320 patients had abandoned leads, and another group about 1,060 patients, did not have abandoned leads. 
These patients were similar in baseline characteristics except that in patients with abandoned leads, the age of the leads is much higher than the ones without abandoned leads. This makes sense since abandoned leads are usually older because they were left behind for a longer period of time. So, for example, the age of the leads in the abandoned group was about 2,291 days compared to 1,550 days in the group with no abandoned leads. The age of the oldest lead was on average 3,670 days in the abandoned leads group compared to only 1,780 days in the group with no abandoned leads. So what does this do? Again, just due to the fact that the leads are much older makes extraction more challenging. In the patients with abandoned leads, the use of more specialized tools was more common. We also found that more tools are needed to extract abandoned lead devices versus leads which were not abandoned.

More importantly the failure to achieve the primary end point which is removal of all lead materials was $15 \%$ in patients with abandoned leads versus only $3.7 \%$ in the group of patients with no abandoned leads. The retention of lead material was much higher at $11.5 \%$ in those with abandoned leads versus only $2.9 \%$ in the patients with no abandoned leads. ${ }^{1}$

\section{Wagner: How does this affect clinical outcomes?}

Wazni: The failure to achieve the clinical endpoint was much higher in those patients with abandoned leads but also the rate of acute complications was much higher in patients with abandoned leads. The rate of having any complication was about $11 \%$ in the abandoned leads group versus $5.6 \%$ in the patients with no abandoned leads, and the rate of major complications was $3.7 \%$ versus $1.4 \%$, which again is much higher in the group of patients with abandoned leads. ${ }^{1}$ All of this tells us that the past decision of leaving a lead behind significantly complicates management of infected devices.

We hope that the findings from this study will encourage physicians and patients to discuss the possible future implications of lead abandonment. I know for a fact that currently this discussion rarely takes place. The patient comes in with a need to upgrade, for example, from a pacemaker to an implantable cardioverter-defibrillator and, most of the time, our patients tell us that they do not remember that they had a discussion regarding abandonment versus extraction. Most of the time, patients are not aware of how many leads they have and that's why we always get X-rays to verify the number of leads present.

The point of the study is not that we want to change the practice completely because I still think the decision has to be individualized. What we are aiming for and hope from the study is that abandonment of the lead should be discussed with the patient and should not be a subject that does not get addressed between the physician and the patient.

Wagner: How will the risk versus risk equation of extracting redundant leads versus the long-term implications of lead abandonment cause physicians to re-evaluate the current practiced strategy?

Wazni: This is a complex issue and I think that on the whole physicians overestimate the risk of extraction, and underestimate the risk of infection later on. It is important for the physician to keep in mind that the risk of extraction is not really that high, especially if they're considering sending the patient to an experienced center where extraction can be performed safely. The other risk is the risk of infection; the reason physicians abandon leads is that they would probably like to defer the risk until later on, but the risk of infection through the years is not trivial either. Patients are living longer and every time there is a device change there is a risk of infection that can be as high as $5 \%$ depending on the series that is quoted. On the whole, the risk of infection is much higher than the risk of extraction. If these devices get infected with the longer implant time, the risk of the extraction itself also becomes much higher. These are very complex issues that should be discussed with the patient before a lead is abandoned. Physicians should not avoid this discussion. It should be part of the consent of abandonment and also extraction. Finally, both the physician and the patient can come up with an informed decision in which both parties understand the implications of either decision.

Wagner: How do specific protocols for patient assessment and care play a key factor in the clinical decision of lead extraction versus lead abandonment?

Wazni: In our practice, we discuss with the patient at length the implications of both abandonment and extraction. Many times the patients will decide to abandon because they're afraid of the immediate risk of extraction. This is understandable but at least we have the discussion.

Wagner: What improvements or advancement do you see on the horizon to further assess the risks of either strategy?

Wazni: I think more electrophysiologists are being trained in extraction and hopefully the procedure itself will become a lot safer. The biggest risk with extraction is perforation at the SVC-RA [superior vena cava-right atrium] junction and when that happens there's not much time for the surgeon to come in and fix the perforation. There is a new device called the Bridge $^{\mathrm{TM}}$ Occlusion Balloon (Spectranetics, Colorado Springs, CO) which is an FDA-approved compliant balloon. This is a balloon that can be placed at the SVC-RA junction in the rare case of SVC-RA perforation hoping to provide more time so as to be able to salvage the patient and have a favorable outcome. Again, although this is a potentially fatal complication, it is very rare. 
Wagner: What are the benefits to patients with the clinical goal of the extraction procedures, which is the elimination of all infected material?

Wazni: The benefit of removing an abandoned lead or a lead that would become abandoned early on is to avoid a long dwell time of this lead. For example, if you have a patient who is 40 years old and they have a need to upgrade from a pacemaker to a defibrillator, if we leave that lead in, there is a potential that this lead could become 30 years old and then get infected. It's much more difficult to remove it when it's 30 years old versus when the lead is only 10 years old. That is the benefit that we're talking about for patients.

\section{Wagner: Are there any additional thoughts you would like to add?}

Wazni: I want to re-emphasize that we should not overestimate the risk of extraction, especially when it is done at centers that do this routinely and the centers of excellence for extraction. Also, we should not underestimate the risk of infection and what abandonment of leads would do in the future, and how abandoned leads eventually complicate the management of these patients in the future.

\section{References}

1. Hussein AA, Khaldoun TG, Martin DO, et al. Cardiac implantable electronic device infections: Added complexity and suboptimal outcomes with previously abandoned leads. JACC Clin Electrophysiol. doi: 10.1016/j.jacep.2016.06.009. 